\section{Sight and comfort: complex procedures in end-stage Stevens-Johnson syndrome}

G Geerling ${ }^{1,2}$, CSC Liu³, JKG Dart', P Sieg², J Herold ${ }^{3}$ and JRO Collin'

\begin{abstract}
Background: We describe our complex surgical techniques in the management of a patient with end-stage ocular surface disease from Stevens-Johnson syndrome.

Methods and results: Her severe discomfort due to absolute ocular dryness in the right eye was successfully treated with submandibular gland autotransplantation. Impending loss of the left eye due to repeated perforation and infection was prevented with a penetrating corneal graft covered by a new modification of a Cutler-Beard-type full-thickness lower lid skin advancement-flap. Visual rehabilitation was achieved by means of osteo-odontokeratoprosthesis.

Conclusion: The procedures described allow the preservation of eyes doomed for enucleation, relief from severe discomfort and rehabilitation from corneal blindness. Due to their complex nature they require the collaborative subspecialist surgical skills of opthalmologists and maxillofacial surgeons. Eye (2003) 17, 89-91. doi:10.1038/

sj.eye. 6700264
\end{abstract}

Keywords: Stevens-Johnson syndrome; blindness; dry eye; rehabilitation; osteo-odonto-keratoprosthesis; submandibular gland transplantation

Stevens-Johnson syndrome is a drug- or infection-induced acute inflammatory reaction of skin and mucous membranes. Progressive conjunctival inflammation and scarring leads to subsequent lid malpositioning, total ocular dryness, surface disease and progressive corneal pannus. Of affected individuals, 35\% experience permanent loss of vision. ${ }^{1}$ Stabilising the ocular surface, reducing the severe discomfort and attempting visual rehabilitation are the major objectives, but any type of corneal graft surgery has an extremely poor prognosis in this situation. ${ }^{2}$ We describe complex surgical techniques to rehabilitate a patient with endstage Stevens-Johnson syndrome by means of a modified Cutler-Beard-type flap,

submandibular gland autotransplantation and osteo-odonto-keratoprosthesis (OOKP).

\section{Case report}

A female caucasian patient suffered from a phenobarbitone-induced Stevens-Johnson syndrome and subsequent progressive ocular surface disease. This disabled her from the age of 29 years to a vision of count fingers (right) and hand motions (left). Despite multiple surgical corrections of lid malposition, the use of gas permeable scleral contact lenses and unpreserved hypromellose $0.3 \%$ every $30 \mathrm{~min}$, she was in permanent severe discomfort due to total ocular dryness. Repeated episodes of left microbial keratitis and perforation were treated with cyanoacrylate glue, one therapeutic lamellar and two penetrating corneal grafts and one heterologous corneal limbal stem cell graft. Epithelial defects recurred, persisted and subsequently became infected with candida. A tarsorraphy could not be performed because of significant lid retraction, so that loss of the left globe was imminent (Figure 1).

To preserve the left eye a fourth corneal graft was performed and immediately covered with a modified Cutler-Beard-type flap. For this a full thickness skin flap was fashioned from the cheek, pulled through under a $6 \mathrm{~mm}$ wide lid margin bridge and fixed to the superior rectus muscle and sclera. Once the flap was vascularised in its bed after 7 months, its base was partially divided and the lower lid reconstructed.
${ }^{1}$ Moorfields Eye Hospital 162 City Road, London, UK

${ }^{2}$ Medizinische Universität Lübeck, Ratzeburger Allee 160, Lübeck, Germany

${ }^{3}$ Sussex Eye Hospital Eastern Road, Brighton, UK

Correspondence:

Dr G Geerling

Department of

Ophthalmology

University Hospital

Lübeck Ratzeburger

Allee 160

23538 Lübeck,

Germany

Tel: +49 4515003090

Fax: +49 4515002671

E-mail: ggeerling@

ophtha.mu-luebeck.de

Received: 19 February 2002 

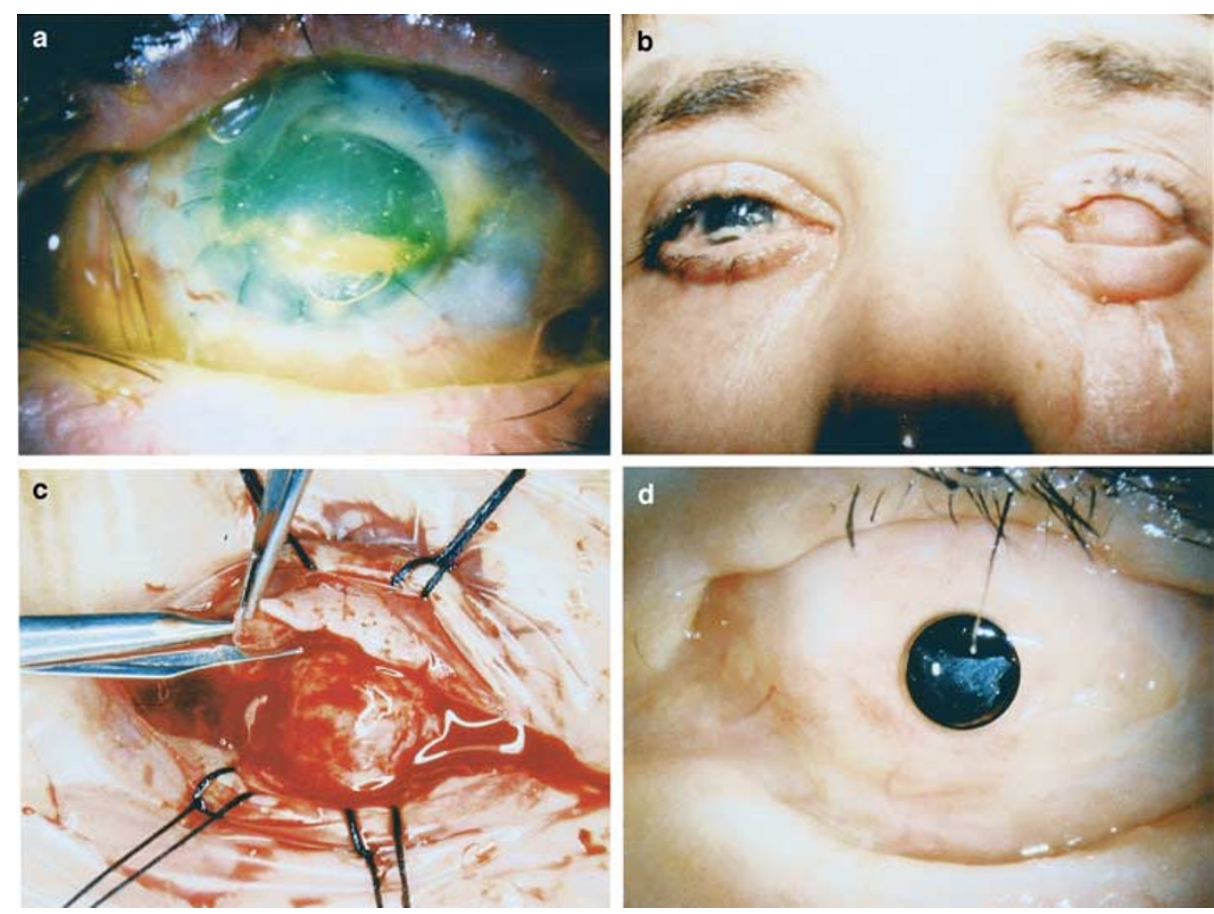

Figure 1 Left eye: (a) Persistent epithelial defect after the third corneal graft despite the use of a scleral contact lens; (b) Seven months after the skin graft was fashioned from the cheek and advanced underneath a bridge of lid margin tissue the flap has been divided and the lower lid reconstructed; (c) Four years later: During OOKP stage 1, the modified Cutler-Beard-type flap is removed. It is found to be extremely well vascularised; (d) Three months after stage 2 .

To relieve the severe dry eye discomfort, a right free submandibular gland autotransplantation to the temporal fossa with microsurgical revascularisation to the temporal artery and vein and implantation of the secretory duct into the superotemporal fornix was performed. ${ }^{3}$ One year postoperatively, Schirmer-Test had improved from 0 to $11 \mathrm{~mm}$. The patient described her preoperative discomfort as 'frequent and severe' and after surgery as 'occasional and mild' and was able to stop the regular application of tear substitutes completely.

Finally, visual rehabilitation was managed in the left eye by means of an OOKP using a refined optical cylinder ${ }^{4}$ and a buccal mucous membrane to cover the osteo-odontal haptic. Three months postoperatively, visual acuity improved to $6 / 6$ and N12 unaided and a visual field with a horizontal span of $100^{\circ}$. After 24 years of registered blindness, she was rehabilitated from disabling pain and blindness and has now worked in a regular job for 2 years.

\section{Comment}

Corneal grafts are the standard surgical approach for corneal ulceration. However, in severe ocular surface disease, they commonly fail ${ }^{5}$ because of compromised corneal wound healing. In addition to limbal stem cell deficiency, the absence of tears reduces epithelial viability due to lack of nutrition, mechanical and antibacterial protection. Thus, epithelial defects recur, persist, progress and become infected. Although new treatment modalities such as amniotic membrane transplantation and autologous serum drops have arrived since this patient's treatment, they are not without failures. ${ }^{6,7}$

Biological flaps and grafts to cover the cornea permanently eliminate pain and the need for topical medication and preserve the eye for later rehabilitation. ${ }^{8}$ To the best of our knowledge, the use of a Cutler-Beardtype skin flap has not been described for this purpose. Its intact primary vascularisation and superior tensile strength provide a major advantage over thin conjunctival flaps or avascular grafts. ${ }^{9,10}$ The disadvantages of the procedure such as poor cosmesis, a most profound reduction of vision and the need to reconstruct the lower lid in a second procedure are justified only in cases such as ours when mucous membrane grafts are likely to fail, especially on the avascular bed of a fresh corneal graft.

Most dry eye patients can be managed with medical treatment. If unpreserved tear substitutes or autologous serum drops fail, surgical tear replacement with 
autologous saliva remains an option. ${ }^{3}$ To avoid gustatory reflex epiphora and to achieve a relatively tear-like composition, a free submandibular gland is currently the only option available. It can largely reduce the symptoms and need for topical tear substitutes in severe dry eyes. Although the procedure involves microvascular anastomosis for revascularisation, with the help of maxillofacial expertise success rates of $77 \%$ have been established. Our current indication is a Schirmer test of $\leq 2 \mathrm{~mm} / 5 \mathrm{~min}$, and severe discomfort, despite frequent use of unpreserved tear substitutes.

OOKP currently is the artificial cornea with the best long-term retention rate. ${ }^{11}$ This is thought to result from the use of a biological material as the haptic (osteoodontal lamina) and a tissue integration time in a subcutaneous pocket prior to implantation. Problems associated with this type of hard optic keratoprosthesis are visual field limitations and the inability to measure intraocular pressure. ${ }^{12}$ However, in view of the poor success of routine medical or surgical treatment, severe ocular surface disease may require complex surgical techniques such as were used in this case, not only to preserve eyes doomed for enucleation, but also to achieve relief from severe discomfort and visual rehabilitation.

\section{Acknowledgements}

This work received its funding from the German Research Council (DFG: Ge 895/4-1), the Special Trustees of Moorfields Eye Hospital and the NHS executive. The views expressed in this publication are those of the authors and not necessarily those of the funding bodies' executives.

\section{References}

1 Howard GM. The Stevens-Johnson syndrome. Ocular prognosis and treatment. Am J Ophthalmol 1963; 55: 893-900.

2 Tsubota K, Satake Y, Ohyama M, Toda I, Takano Y, Ono M et al. Surgical reconstruction of the ocular surface in advanced ocular cicatricial pemphigoid and StevensJohnson syndrome [see comments]. Am J Ophthalmol 1996; 122(1): 38-52.

3 Geerling G, Sieg P, Bastian GO, Laqua H. Transplantation of the autologous submandibular gland for most severe cases of keratoconjunctivitis sicca. Ophthalmology 1998; 105(2): 327-335.

4 Hull CC, Liu CSC. Towards an improved visual field in the osteo-odonto keratoprosthesis. Ophthalmic Physiol Opt 1998; 18: 382 .

5 Tugal-Tutkun I, Akova YA, Foster CS. Penetrating keratoplasty in cicatrizing conjunctival diseases. Ophthalmology 1995; 102(4): 576-585.

6 Chiou AG, Florakis GJ, Kazim M. Management of conjunctival cicatrizing diseases and severe ocular surface dysfunction. Surv Ophthalmol 1998; 43(1): 19-46.

7 Poon AJ, Geerling G, Dart JKG GEF. Autologous serum eyedrops for dry eyes and epithelial defects: clinical and in-vitro toxicity studies. Br J Ophthalmol, 2001; 85: 1188-1197.

8 Mauriello Jr JA, Pokorny K. Use of split-thickness dermal grafts to repair corneal and scleral defects - a study of 10 patients [see comments]. Br J Ophthalmol 1993; 77(6): 327-331.

9 Heiligenhaus A, Shore JW, Rubin PA, Foster CS. Long-term results of mucous membrane grafting in ocular cicatricial pemphigoid. Implications for patient selection and surgical considerations. Ophthalmology 1993; 100(9): 1283-1288.

10 Alino AM, Perry HD, Kanellopoulos AJ, Donnenfeld ED, Rahn EK. Conjunctival flaps [see comments]. Ophthalmology 1998; 105(6): 1120-1123.

11 Marchi V, Ricci R, Pecorella I, Ciardi A, Di Tondo U. Osteoodonto-keratoprosthesis. Cornea 1994; 13(2): 125-130.

12 Dohlman $\mathrm{CH}$, Terada $\mathrm{H}$. Keratoprosthesis in pemphigoid and Stevens-Johnson syndrome. Adv Exp Med Biol 1998; 438: 1021-1025. 